\section{Nachweis einer Katalase-Hemmung durch Formaldehyd in vitro}

\section{B. A. F. Stumm-Tegethoff und A. H.M. van Der Loo}

Genetisches Institut der Universität Nijmegen (Niederlande)

(Z. Naturforschg. 24 b, 657-658 [1969] ; eingegangen am 7. Januar 1969)

Mutationsversuche an Drosophila melanogaster ${ }^{1,2}$ machen wahrscheinlich, daß die mutagene Wirkung von Formaldehyd in saurem Milieu sowie einiger Säuren, auf Katalase-Hemmung beruht. Eine Folge dieser Hemmung wäre eine Anhäufung von $\mathrm{H}_{2} \mathrm{O}_{2}$ und von Peroxyden, deren Mutagenität schon früher beschrieben wurde $^{3-6}$. Um diese Arbeitshypothese zu stützen, haben wir die hier wiedergegebenen in-vitro-Experimente durchgeführt.

In Anlehnung an bekannte Katalase-Nachweismethoden ${ }^{7}$ wurde die Katalase-Aktivität spektrophotometrisch bestimmt. Bei $240 \mathrm{~m} \mu$ wurde die Abnahme der optischen Dichte als Folge des Substratabbaues durch Katalase gemessen. Als Substrat diente $0,314 \mathrm{M} \mathrm{H}_{2} \mathrm{O}_{2}-\mathrm{Lö}-$ sung in $\mathrm{S}$ ör en sen-Puffer, $\mathrm{pH} 7,02$, wovon $3 \mathrm{ml}$ in

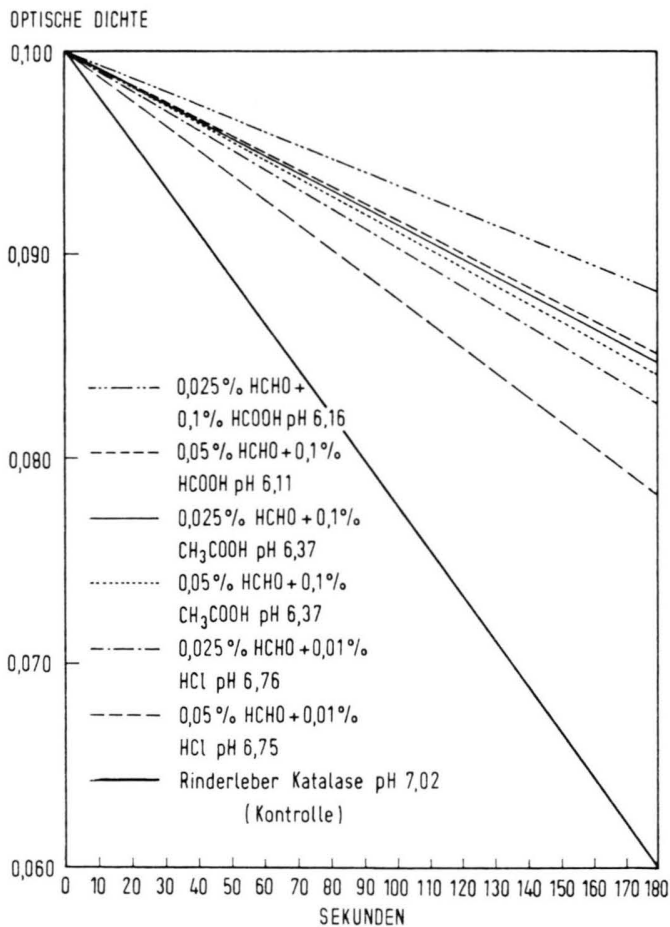

Schema 1. Darstellung der Mittelwerte (ohne Berücksichtigung der Abweichung) der Extinktionsabnahme/10 sec. Den berechneten Werten liegen mindestens 3 Versuchsserien zu grunde.

1 B. Stumm-Tegethoff, Naturwissenschaften 51, 646 [1964]

2 Publikation in Vorbereitung.

3 F. H. Sobels, Z. ind. Abst.- u. Vererblehre 86, 399 [1955].

${ }^{4}$ F. H. Sobels, Nature [London] 177, 979 [1956]. die Meßküvetten gegeben wurden. Katalase aus Rinderleber der $\mathrm{Fa}$. Boehringer mit einer spezifischen Aktivität von $39000 \mathrm{U} / \mathrm{mg}$ wurde so verdünnt, daß 4,75 U/ $\mu \mathrm{l}$ in den Versuch genommen wurden. Die Abnahme der optischen Dichte infolge der Reduktion von $\mathrm{H}_{2} \mathrm{O}_{2}$ durch die zugefügte Katalase wurde in Abständen von $10 \mathrm{sec}$ 3 min lang gemessen. Die Küvettentemperatur des UV. Spektrophotometers (Zeiss, PMQ II) betrug konstant $20{ }^{\circ} \mathrm{C}\left( \pm 0,2{ }^{\circ} \mathrm{C}\right)$.

Die Resultate sind Mittelwerte aus mindestens 3 Versuchsreihen. Die Konzentration der Substanzen, deren Einfluß auf die Katalase-Aktivität zu untersuchen war, wurde so gewählt, daß sie mit der der im Mutationsversuch verwendeten Lösungen übereinstimmte. Die Stammlösungen für Formaldehyd, Ameisen-, Essig- und Salzsäure waren so eingestellt, da $\beta$ nach Hinzufügen der Substanzen zu den $3 \mathrm{ml} \mathrm{H}_{2} \mathrm{O}_{2}$-Lösung die Endkonzentration in der Küvette $0,05 \%, 0,025 \%, 0,1 \%, 0,1 \%$ bzw. $0,01 \%$ betrug. Zur Herstellung dieser Stamm-

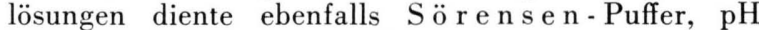
7,02 als Verdünnungsmittel. Die $\mathrm{H}_{2} \mathrm{O}_{2}$-Lösung wurde mit den auf Hemmwirkung zu untersuchenden Substan-

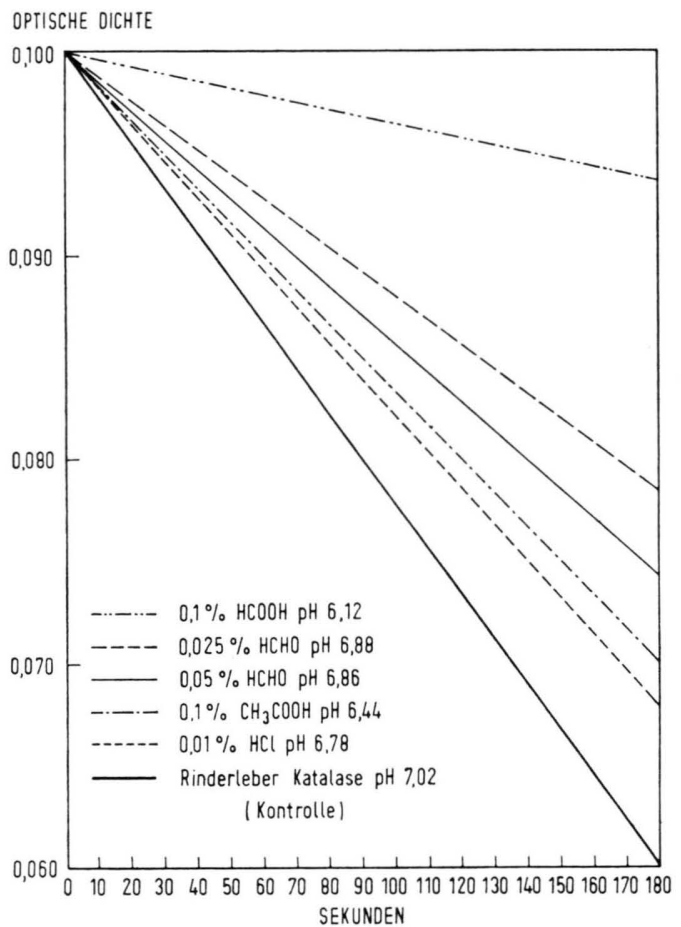

Schema 2. Darstellung der Mittelwerte (ohne Berücksichtigung der Abweichung) der Extinktionsabnahme/10 sec. Den berechneten Werten liegen mindestens 3 Versuchsreihen zugrunde.

5 F. H. Sobels and J. W. J. M. Simons, Z. ind. Abst.- u. Vererblehre 87, 735 [1956].

${ }^{6}$ F. H. Sobels, Radiat. Res. Suppl. 3, 171 [1963].

7 H. LücK, in: Methoden der enzymatischen Analyse, Verlag Chemie, Weinheim/Bergstraße 1962. 
zen versetzt und gut gemischt, dann 1. der $\mathrm{pH}$-Wert und 2. die Extinktion gemessen und sofort die Katalase zugefügt.

Die Ergebnisse sind in Schemata 1 und 2 zusammengestellt und geben den Einfluß der untersuchten Stoffe auf die Katalase-Aktivität wieder.

Alle untersuchten Agenzien hemmen die Katalase.

AgNer und THEORELL ${ }^{8}$ weisen eine Katalase-Hemmung durch Anionen nach. LücK ${ }^{9}$ findet, daß die Abhängigkeit vom $\mathrm{pH}$-Wert Dissoziationsgrad und Konstitution der Säure und die Katalase-Hemmung entscheiden. Das Aktivitätsoptimum der Katalase liegt bei pH 7 . Wir wählten daher $\mathrm{pH}$ 7,02 für den Kontrollversuch. Inwieweit die sich ergebende Zunahme der H-IonenKonzentration bei Zugabe von Säuren oder anderen Substanzen und die damit verbundene Herabsetzung des $\mathrm{pH}$-Wertes oder andererseits der Dissoziationsgrad der Säure für die Katalase-Hemmung anzusprechen sind, braucht durch unsere Versuche nicht entschieden zu werden. Die Tatsache des Auftretens einer KatalaseHemmung allein genügt, um eine Anhäufung von $\mathrm{H}_{2} \mathrm{O}_{2}$ zu bewirken, die mutagene Folgen haben kann. Obwohl LÜCK ${ }^{10}$ mit viel geringeren Konzentrationen (für $\mathrm{HCOOH}$ : $0,002 \mathrm{M}$ ) als wir (für $\mathrm{HCOOH}: 0,265 \mathrm{M}$ ) und feststehendem $\mathrm{pH}$-Wert $(4,5)$ arbeitet, findet auch er, daß die Ameisensäure von allen durch ihn getesteten Stoffen die stärkste Katalase-Hemmung aufweist. Für

8 K. Agner and H. Theorell, Arch. Biochem. 10, 321 [1946]. unsere Versuche war die Festlegung auf einen bestimmten $\mathrm{pH}$-Wert nicht von Bedeutung, da sich bei allen aus der Zusammensetzung der Reaktionsgemische ergebenden pH-Werten eine mehr oder weniger starke Katalase-Hemmung zeigte. Mit LücK stimmen wir auch darin überein, daß in bezug auf Essigsäure eine Fehlinterpretation der Ergebnisse möglich ist, da Essigsäure durch das vorhandene $\mathrm{H}_{2} \mathrm{O}_{2}$ oxydiert wird. Dies würde die geringere Hemmwirkung von $\mathrm{CH}_{3} \mathrm{COOH}$ gegenüber $\mathrm{HCOOH}$ erklären.

Die vorliegenden Versuche bestätigen eine KatalaseHemmung durch Formaldehyd bei niedrigen $\mathrm{pH}$-Werten; bei $\mathrm{pH}$-Werten $>7$ bleibt Formaldehyd ohne nennenswerten Einfluß auf die Katalase. Durch Zugabe von Säuren, die jede bereits für sich eine Katalase-Hemmung bewirken, wobei $\mathrm{HCOOH}$ die stärkste, HCl die geringste Hemmung zeigt, wird die Katalase-Hemmung durch Formaldehyd verstärkt, jedoch kann nicht von einer additiven Wirkung der Substanzen gesprochen werden. Die Ergebnisse stimmen weitgehend überein mit dem Maß der Mutationsempfindlichkeit von Drosophila melanogaster für diese Substanzen ${ }^{1,2}$ und verstärken daher den Eindruck, daß die Katalase-Hemmung, die zu einer Anhäufung von $\mathrm{H}_{2} \mathrm{O}_{2}$ und damit zur Entstehung von mutagenen Peroxyden führt, durch Formaldehyd bei niedrigem $\mathrm{pH}$-Wert bzw. Säuren hervorgerufen wird.

9 H. Lück, Naturwissenschaften 44, 423 [1957].

10 H. LücK, Biochem. Z. 328, 411 [1957]. 